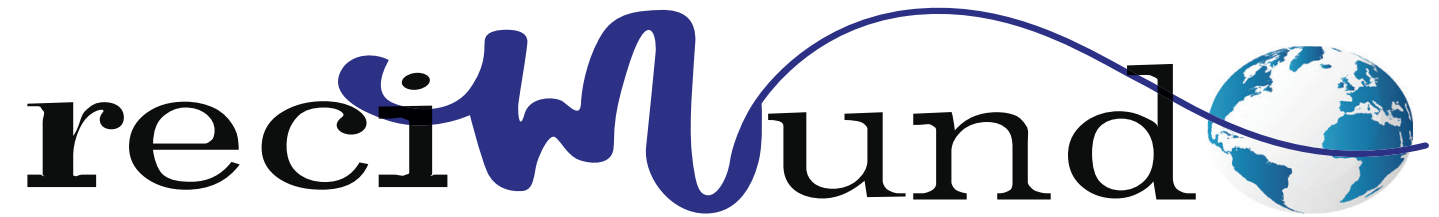

Revista Científica Mundo de la Investigación y el Conocimiento

DOI: 10.26820/recimundo/4.(1).enero.2020.267-276

URL: http://recimundo.com/index.php/es/article/view/741

EDITORIAL: Saberes del Conocimiento

REVISTA: RECIMUNDO

ISSN: 2588-073X

TIPO DE INVESTIGACIÓN: Artículo de Revisión

Código UNESCO: 32 Ciencias Médicas

PAGINAS: 267-276

\title{
Luxación acromioclavicular: tratamiento conservador
}

\author{
Acromioclavicular dislocation: \\ conservative treatment
}

\section{Luxação acromioclavicular: tratamento conservador}

Verónica Teresa León Portilla; Janio Luis Pico Segarra2; Maite Guisella Santillán Arias³

RECIBIDO: 18/09/2019 ACEPTADO: 29/10/2019 PUBLICADO: 31/01/2020

1. Médico; Universidad de Guayaquil; Guayaquil, Ecuador; vero_portilla@hotmail.com; (D) https://orcid.org/0000-0002-77034183

2. Médico; Universidad de Guayaquil; Guayaquil, Ecuador; janiopico@hotmail.com; (D) https://orcid.org/0000-0002-29049911

3. Médico; Investigador Independiente; Guayaquil, Ecuador; maitesantillan92@gmail.com; (DD https://orcid.org/0000-00021366-8187

CORRESPONDENCIA

Verónica Teresa León Portilla

vero_portilla@hotmail.com

Guayaquil, Ecuador

() RECIMUNDO; Editorial Saberes del Conocimiento, 2020 


\section{RESUMEN}

La luxación de la articulación acromioclavicular es una lesión traumática donde se produce una rotura de las estructuras estabilizadoras de la articulación: Ios ligamentos acromioclaviculares y coracoclaviculares, y de la cápsula. El extremo acromial de la clavícula se eleva a un nivel superior al del acromion ocasionando una deformidad perceptible y un descenso del hombro lesionado.Se logra comprender entonces que, una luxación es cuando un hueso, que forma parte de una articulación, se sale de la misma, produciendo dolor e incapacidad funcional. Esta lesión, se debe de manera general a caídas sobre el hombro, ameritando si es leve de un tratamiento conservador; no obstante, si la lesión de los ligamentos es más compleja se aconseja pasar por el quirófano. Entre los deportes de mayor riesgo que producen esta patología se logra citar: los deportes de contacto en los que con un golpe producen en el acromion una tracción de los ligamentos acromioclaviculares, en caídas sobre el codo o el brazo, la cabeza humeral golpea contra el acromion y desplaza la escapula hacia arriba en relación a la clavícula, al romperse los ligamentos por el impacto de caídas sobre el hombro empuja la escápula para abajo. De manera general, esta lesión es causada cuando la clavícula, al estar ligada a la caja torácica no puede acompañar el movimiento de la escápula por lo que los ligamentos de la articulación acromio clavicular se comienza a romper y la articulación empieza a separarse, esto lleva a señalar que, las luxaciones acromoclavicular se pueden clasificar en leve, moderada y grave dependiendo de que ligamentos son los distendidos o rotos. El tratamiento de la Luxación acromioclavicular actual permite una rápida recuperación del hombro a su actividad habitual. En los casos de lesión desplazada se aconseja la estabilización quirúrgica.

Palabras clave: Luxación Acromioclavicular, Llesiones Traumáticas, Ligamentos Acromioclaviculares, Coraclaviculares y de Cápsula.

\section{ABSTRACT}

The dislocation of the acromioclavicular joint is a traumatic injury where there is a rupture of the stabilizing structures of the joint: the acromioclavicular and coracoclavicular ligaments, and of the capsule. The acromial end of the clavicle rises to a level higher than that of the acromion causing a noticeable deformity and a lowering of the injured shoulder. It is then understood that a dislocation is when a bone, which is part of a joint, leaves the same, producing pain and functional disability. This injury is generally due to falls on the shoulder, meriting if it is mild from a conservative treatment; however, if the ligament injury is more complex, it is advisable to go through the operating room. Among the most risky sports that produce this pathology, it is possible to mention: contact sports in which, with a blow, they cause acromioclavicular ligament traction in the acromion, in falls on the elbow or arm, the humeral head strikes against the acromion and shifts the scapula upwards in relation to the clavicle, when the ligaments rupture due to the impact of falls on the shoulder pushes the scapula down. In general, this lesion is caused when the clavicle, being linked to the rib cage, cannot accompany the movement of the scapula so that the ligaments of the clavicular acromium joint begin to break and the joint begins to separate, this leads Note that acromoclavicular dislocations can be classified as mild, moderate and severe depending on which ligaments are distended or broken. The treatment of current acromioclavicular dislocation allows a rapid recovery of the shoulder to its usual activity. In cases of displaced injury, surgical stabilization is advised.

Keywords: Acromioclavicular Dislocation, Traumatic Injuries, Acromioclavicular, Coraclavicular and Capsule Ligaments.

\section{RESUMO}

A luxação da articulação acromioclavicular é uma lesão traumática onde ocorre uma ruptura das estruturas estabilizadoras da articulação: os ligamentos acromioclavicular e coracoclavicular e da cápsula. A extremidade acromial da clavícula sobe para um nível superior ao do acrômio, causando uma deformidade perceptível e uma diminuição do ombro lesionado. Entende-se então que uma luxação ocorre quando um osso, que faz parte de uma articulação, deixa o mesmo, produzindo dor e incapacidade funcional. Essa lesão geralmente é devida a quedas no ombro, merecendo ser leve de um tratamento conservador; no entanto, se a lesão ligamentar for mais complexa, é aconselhável passar pela sala de operações. Entre os esportes mais arriscados que produzem essa patologia, é possível mencionar: esportes de contato nos quais, com um golpe, causam tração do ligamento acromioclavicular no acrômio, em quedas no cotovelo ou braço, a cabeça do úmero bate contra o acrômio e move a escápula para cima em relação à clavícula, quando os ligamentos se rompem devido ao impacto de quedas no ombro empurram a escápula para baixo. Em geral, essa lesão é causada quando a clavícula, ligada à caixa torácica, não pode acompanhar o movimento da escápula, de modo que os ligamentos da articulação do acrômio clavicular começam a se romper e a articulação começa a se separar, o que leva a notar que as luxações acromoclaviculares pode ser classificado como leve, moderado e grave, dependendo de quais ligamentos estão distendidos ou quebrados. O tratamento da atual luxação acromioclavicular permite uma rápida recuperação do ombro à sua atividade habitual. Nos casos de lesão deslocada, recomenda-se a estabilização cirúrgica.

Palavras-chave: Luxação Acromioclavicular, Lesões Traumáticas, Ligamentos Acromioclavicular, Coracoclavicular e Cápsula. 


\section{Introducción}

Los pacientes con lesiones acromioclaviculares grado III suponen un reto terapéutico para el cirujano de hombro. Aunque algunos autores se apuntan a la tendencia habitual de tratar las lesiones musculo esqueléticas del hombro de manera cada vez más agresiva (es decir, quirúrgicamente), la realidad es que estas lesiones se pueden manejar de forma conservadora en su gran mayoría. (Díaz Heredia, Ruiz Díaz, \& Valencia Mora , 2015)

En primer lugar, los datos de distintos meta análisis recientes sugieren que esta es la mejor opción; segundo, estos mismos meta análisis refrendan la bondad del tratamiento conservador en pacientes muy jóvenes; tercero, los pacientes con altos requerimientos funcionales también obtienen buenos resultados con el tratamiento conservador; cuarto, desde un punto de vista económico el tratamiento conservador es más ventajoso; quinto, la deformidad residual, aunque persiste, no es un problema a largo plazo; sexto, la aparición de disfunción escapulotorácica puede corregirse con una terapia física adecuada.

En séptimo lugar séptimo, la artrosis aparece en la articulación a largo plazo se opere o no, y por último, cuando infrecuentemente algún paciente requiere ser intervenido de forma diferida, los resultados son similares a los de la cirugía primaria. Es notable entonces destacar que, la articulación acromioclavicular se localiza en la parte superior del hombro. La misma es localizada en la articulación entre la clavícula y el acromion (una proyección del omóplato que se encuentra en el extremo del hombro). (Sapunar \& Velasco, 2000)

La luxación de la articulación acromioclavicular es una de las lesiones de hombro tratada con mayor frecuencia en la población deportista activa. Es una lesión común del hombro en ciclistas y deportistas de contacto como futbolistas, boxeadores y practicantes de artes marciales. De esta mane- ra, se logra señalar que, la luxación ocurre habitualmente en hombres jóvenes que se caen y sufren un impacto directo en la parte superior del hombro. (Gutiérrez Blanco, Sánchez Bejarano, \& Reyes Casales, 2001)

De igual forma, hay que puntualizar que la mayoría de las luxaciones acromioclaviculares se tratan sin cirugía, especialmente las que presentan una separación menos grave entre los huesos. (Tamaoki, Lenza, \& Matsunaga, 2017, pág. 13). El tratamiento no quirúrgico o conservador implica la inmovilización del brazo. Sin embargo, el resultado puede ser menos satisfactorio cuando ocurre una luxación más grave. Los tratamientos quirúrgicos involucran el reposicionamiento de las partes articulares y la reparación o reconstrucción de los ligamentos rotos.

Entiéndase a través de esto que, no existe un consenso general sobre cuál es el tratamiento más adecuado para el manejo de las lesiones acromioclaviculares. El objetivo del tratamiento de estas lesiones es obtener un hombro no doloroso con una recuperación completa de la movilidad y de la fuerza. Los defensores del tratamiento conservador "sostienen que se obtienen buenos resultados funcionales sin dolor con una tasa baja de complicaciones, aunque en algunos pacientes persisten inestabilidad y dolor". (Tamaoki, Lenza, \& Matsunaga, 2017, pág. 13)

Por otro lado, el tratamiento quirúrgico evitaría la inestabilidad y mejoraría el resultado cosmético. Sin embargo, se han descrito numerosos episodios adversos asociados al tratamiento quirúrgico y un mayor tiempo de recuperación. Por su parte, según (Tamaoki, Lenza, \& Matsunaga, 2017, pág. 13) "el tratamiento conservador incluye un periodo de inmovilización de 2 semanas con un cabestrillo, el uso de hielo local, antiinflamatorios y analgésicos". Se permiten desde el principio ejercicios de movilización pasivos y el uso del brazo por debajo del hombro limitados por el dolor.

En la tercera semana se inicia un protocolo

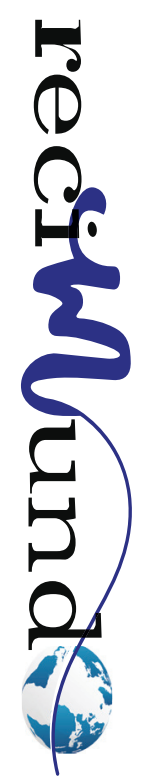


de rehabilitación, con especial interés en el fortalecimiento de la articulación escapulotorácica para mantener la estabilización de la escápula, ya que con la lesión se produce una disrupción de la unidad suspensoria del hombro. Finalmente, a criterio de (Tamaoki, Lenza, \& Matsunaga, 2017, pág. 14), "se permite la reincorporación a la actividad deportiva una vez recuperada una función del hombro no dolorosa". De allí, que la opción quirúrgica se plantee si tras 12 semanas de tratamiento conservador persiste sintomatología dolorosa o limitación funcional.

Compréndase con esto que, el tratamiento conservador de las lesiones acromioclaviculares grado III a criterio de (Kayap, 2016, pág. 17), "representa la mejor alternativa en base a la evidencia existente por su alta tasa de buenos resultados, el menor tiempo de recuperación"; de ahí que, sus escasas complicaciones y la posibilidad de una cirugía diferida en caso de mala evolución, con resultados equiparables a los de la cirugía aguda.

\section{Metodología}

\section{Fuentes documentales}

El análisis correspondiente a las características que predomina en el tema seleccionado, llevan a incluir diferentes fuentes documentales encargadas de darle el respectivo apoyo y en ese sentido cumplir con la valoración de los hechos a fin de generar nuevos criterios que sirven de referencia a otros procesos investigativos. Para (Arias, 2010), las fuentes documentales incorporadas en la investigación documental o bibliográfica, "representa la suma de materiales sistemáticos que son revisados en forma rigurosa y profunda para llegar a un análisis del fenómeno". (p.41). Por lo tanto, se procedió a cumplir con la realización de una lectura previa determinada por encontrar aquellos aspectos estrechamente vinculados con la "LUXACIÓN ACROMIOCLAVICULAR: TRATAMIENTO CONSERVADOR" para luego explicar mediante un desarrollo las respectivas apreciaciones generales de importan- cia.

Técnicas para la Recolección de la Información

La conducción de la investigación para ser realizada en función a las particularidades que determinan a los estudios documentales, tiene como fin el desarrollo de un conjunto de acciones encargadas de llevar a la selección de técnicas estrechamente vinculadas con las características del estudio. En tal sentido, (Arias Ob cit) refiere, que es "una técnica particular para aportar ayuda a los procedimientos de selección de las ideas primarias y secundarias". (p. 71).

Por ello, se procedió a la utilización del subrayado, resúmenes, fichaje, como parte básica para la revisión y selección de los documentos que presentan el contenido teórico. Es decir, que mediante su aplicación de estas técnicas se pudo llegar a recoger informaciones en cuanto a la revisión bibliográfica de los diversos elementos encargados de orientar el proceso de investigación. Tal como lo expresa, (Arias Ob cit) "las técnicas documentales proporcionan las herramientas esenciales y determinantes para responder a los objetivos formulados y llegar a resultados efectivos" (p. 58). Es decir, para responder con eficiencia a las necesidades investigativas, se introdujeron como técnica de recolección el método inductivo, que hizo posible llevar a cabo una valoración de los hechos de forma particular para llegar a la explicación desde una visión general.

Asimismo, se emplearon las técnicas de análisis de información para la realización de la investigación que fue ejecutada bajo la dinámica de aplicar diversos elementos encargados de determinar el camino a recorrer por el estudio, según, (Arias, Ob cit) las técnicas de procesamiento de datos en los estudios documentales "son las encargadas de ofrecer al investigador la visión o pasos que debe cumplir durante su ejercicio, cada una de ellas debe estar en correspondencia con el nivel a emplear" ( $p$. 
123). Esto indica, que para llevar a cabo el procesamiento de los datos obtenidos, es necesario establecer las técnicas que serán seleccionadas, destacándose en este caso, de manera particular: fichas de resumen, textual, registros descriptivos entre otros, los mismos se deben ajustar al nivel que ha sido seleccionado.

\section{Resultados}

\section{¿Qué es la Luxación Acromioclavicular?}

El hombro es una articulación compleja formada por varias estructuras todas unidas entre ellas para permitir que sea la articulación con más movilidad del cuerpo. Una de estas articulaciones según (Kayap, 2016, pág. 19) "es la acromioclavicular, que une la clavícula con el acromion". He ahí, que la articulación acromioclavicular se encuentra estabilizada por varios ligamentos que permiten mantener su posición. Se conocen como los ligamentos coracoclaviculares y los ligamentos acromioclaviculares.

La luxación acromioclavicular se produce tras una caída accidental con contusión sobre el propio hombro. Para (Kayap, 2016, pág. 17), "la contusión directa sobre el acromion produce un efecto palanca que lesiona los ligamentos estabilizadores de la articulación acromioclavicular", lo que en su efecto, produce lo que se conoce como luxación acromioclavicular. Esta lesión es muy frecuente en ciclistas, en jugadores de rugby y futbol americano.

El tratamiento a seguir, tras producirse una luxación acromioclavicular se basa en realizar una inmovilización con un sling o cabestrillo durante unos 10-15 días.

En el caso de que se realice un tratamiento quirúrgico o tratamiento conservador según (Kayap, 2016, pág. 17), "es recomendable empezar la movilización pasiva del hombro mediante ejercicios pendulares sobre las 2 semanas".

Posteriormente se pueden realizar ejercicios de movilidad asistida y finalmente ejercicios de movilidad activa. La pauta de ejercicios de tratamiento de la luxación acromioclavicular es muy similar a la pauta de recuperación de la inestabilidad de hombro, pero con menos tiempo de inmovilización inicial. En base a esto, (Kayap, 2016, pág. 20), refiere "los síntomas más frecuente de la luxación acromioclavicular son el dolor en la zona anterior del hombro y la deformidad del mismo". Esto lleva a apreciar el ascenso de la punta de la clavícula en casos más severos de luxación acromioclavicular.

El diagnostico es principalmente clínico, el dolor en al zona anterior del hombro, sobre la articulación acromioclavicuilar tras una contusión es muy típico. Para ello, según (Kayap, 2016, pág. 17) "es recomendable realizar una radiografía para valorar la separación entre el acromion y la clavícula". Esto lleva a entender que, la clasificación de la luxación acromioclavicular denominada clasificación de Rockwood se establece según el grado de separación radiografía. Existen 6 tipos de luxación acromioclavicular que van de menos grado de desplazamientos los tipos I II y III hasta los tipos que presentan una separación muy severa como los tipos IV V y VI.

Asimismo, "la lesión del hombro luxación acromioclavicular tiene en general un pronostico muy bueno de recuperación y de retorno al deporte". (Kayap, 2016, pág. 17) Conforme a esto, es de vital importancia señalar que, no todas las luxaciones acromioclaviculares evolucionan bien, en ocasiones existen pacientes que desarrollan una artrosis acromioclavicular que precisa su tratamiento. En otros pacientes la deformidad residual que queda es importante y requiere de una intervención quirúrgica para corregir este problema.

Por tal motivo, el tratamiento más habitual para este tipo de lesiones de luxación crónica acromioclavicular, es la cirugía. Se realiza una resección de la parte distal de la clavícula y se estabiliza la clavícula con una plastia ligamentosa entre la coracoides y la

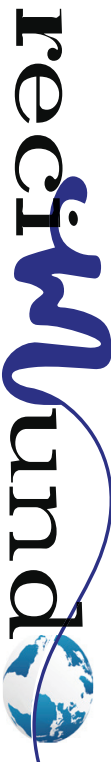


clavícula.

Los resultados del tratamiento de las lesiones crónicas de luxación acromioclavicular son buenos. (Kayap, 2016, pág. 20).

\section{Tratamiento de la luxación acromioclavi- cular}

El tratamiento inicial más recomendado para una luxación acromioclavicular es el reposo, el uso de un sling para inmovilizar el hombro y los analgésicos-antiiflamatorios. Como cualquier lesión de hombro, "los primeros dias son dolorosos y la persona tiene varias dificultades para dormir". (Korsten, 2017, pág. 40) Por ello, una vez que se haya establecido el diagnóstico y la clasificación de la luxación acromioclavicular, se realiza el tratamiento definitivo.

El tratamiento de la luxación acromioclavicular de los tipos I y II es conservador, no se recomienda la cirugía. Se realiza una inmovilización del hombro durante unas 3 semanas y posteriormente se inicia un periodo de recuperación de la movilidad del hombro. Posteriormente se recupera la propiocepción y la fuerza. Por otra parte, el tratamiento de la luxación acromioclavicular de los tipos IV V VI es siempre quirúrgico, se recomienda una reducción y una estabilización de la articulación. (Astudillo Valenzuela \& Yoma Galleguillos, 2009)

La luxación tipo III es la que tiene un tratamiento más controvertido. Diferentes estudios han comparado el tratamiento conservador con el tratamiento quirúrgico, los resultados muestran que los pacientes se recuperan un poco antes sin realizar una intervención, pero queda una cierta deformidad en el hombro. De ahí, que sea importante valorar que a veces es difícil diferenciar radiográficamente una luxación acromioclavicular tipo III con la tipo V. (Korsten, 2017, pág. 37)

En los tipos IV V y VI, y en algunos casos de tipo III se recomienda realizar un tratamiento mediante cirugía. Los objetivos del tratamiento quirúrgico en la luxación acromioclavicular son la reducción de la articulación y la estabilización ósea. La necesidad de cirugía de la lesión. Para (Korsten, 2017, pág. 35) como se había sugerido, "se recomendará un tratamiento conservador para lesiones de grado leves, centrado en analgésicos que ayuden a calmar el dolor y el reposo durante unas semanas con cabestrillo". Esto se hará, posterior a las semanas de recuperación se aconseja seguir un programa de rehabilitación por un fisioterapeuta u otro profesional.

También, hay que tener presente que en pacientes que tienen una exigencia funcional mayor del miembro superior, el tratamiento quirúrgico es lo indicado. Para lesiones más graves y pacientes con una gran demanda física y funcional de la zona afectada se le recomendaría una cirugía para la luxación acromioclavicular. (Korsten, 2017, pág. 37) Para el tratamiento quirúrgico la técnica más utilizada es la inserción de agujas de kirshner, sin necesidad de corte, y el injerto de tendones para las lesiones más graves. Existe una amplia variedad de técnicas que se adaptan al tipo de lesión, gravedad de la luxación y nivel funcional del miembro.

\section{Anatomía}

La articulación acromioclavicular se encuentra conformada por 2 huesos, los mismos se describen de la siguiente forma:

- El acromion, que es una protuberancia que forma parte de la escápula, uno de los huesos más importantes de la extremidad superior.

- La clavícula es un hueso que se encuentra en el cuello que se extiende desde la articulación esternoclavicular, en el esternón, hasta la articulación acromioclavicular, cerca del hombro. (Korsten, 2017, pág. 41)

Esta articulación esta rodeada por varios ligamentos, conocidos como ligamento acromioclavicular y coracoclavicular, los cuales 
son responsables de proporcionar la estabilidad necesaria para que los huesos que forman la articulación AC no salgan de su sitio. Por su parte, la articulación AC ayuda a dar estabilidad al hombro, por lo que su lesión podría producir dolor y dificultades para el movimiento normal del hombro. (Slullitel, Galán, \& Della, 2016)

\section{Mecanismo de lesión}

La causa más común de una luxación $A C$ es una caída con un traumatismo directo sobre el hombro. Si la fuerza del traumatismo es muy severa, los ligamentos que se encuentran alrededor de la articulación AC pueden romperse. Esto provoca la "separación" de la clavícula y el acromion, causando una luxación AC. (Korsten, 2017, pág. 42)

\section{Gravedad de la lesión}

La luxación AC, es aquella que puede producir diversos grados de lesión, siendo los mismos los siguientes:

- Una luxación grado I de la articulación AC implica un esguince de los ligamentos de la articulación AC sin movimiento la clavícula que se ve normal en las radiografías.

- Una luxación grado II produce un desgarro del ligamento AC y desgarra ligeramente el ligamento coracoclavicular (CC), produciendo que la clavícula este moderadamente fuera de su alineación normal.

- Una luxación grado III rompe completamente los ambos ligamentos AC y CC, produciendo una separación y deformidad muy importante. (Tamaoki, Lenza, \& Matsunaga, 2017, pág. 13)

\section{Tratamiento conservador}

Muchos de los pacientes, presentan mejoría total de sus síntomas con tratamiento conservador, el cual consiste en:

- El uso de cabestrillo, para inmovilizar el hombro afectado y permitir una recupe- ración adecuada.

- Uso de bolsas de hielo por 15 minutos para disminuir la inflamación.

- Uso de analgésicos como el ibuprofeno, que pueden ayudar a controlar el dolor.

- La mayoría de la gente vuelve a la función casi completa después de la lesión, incluso si queda deformidad residual y significativa en la articulación AC. (Korsten, 2017, pág. 42)

\section{Tratamiento quirúrgico}

La cirugía puede ser necesaria si el dolor o la deformidad son severos y persistentes. La cirugía consiste en:

- Su ortopedista podría cortar el extremo de clavícula que se articula con el acromion, esto para impedir que exista contacto contra el acromion y evitar el dolor.

- Cuando hay deformidad significativa, la reconstrucción de los ligamentos coracoclaviculares es útil. (Korsten, 2017, pág. 43)

\section{Rehabilitación}

Independiente de si es tratado de forma conservadora o con cirugía, su hombro lesionado requerirá de rehabilitación para restaurar los rangos del movimiento, la fuerza y la flexibilidad lo mayormente posible 


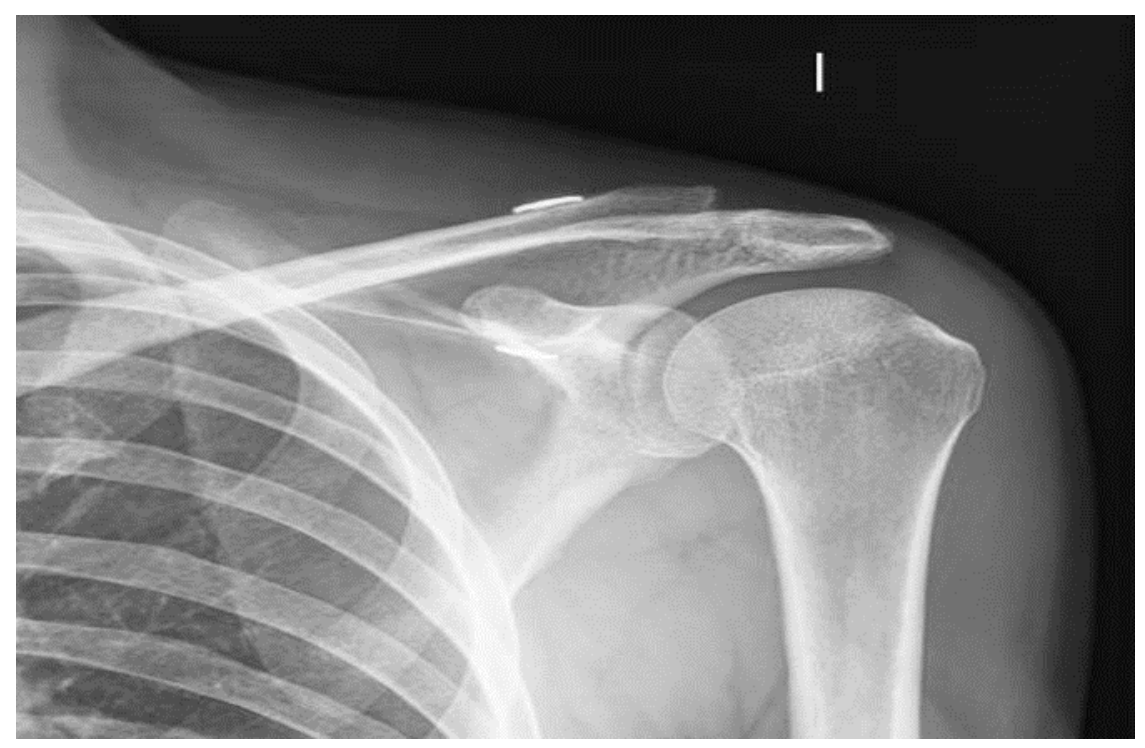

Figura 1. Articulación Acromioclavicular

Fuente: (Korsten, 2017)

Lo señalado en la imagen expuesta lleva a establecer que, un hombro dislocado se suele poder identificar durante una exploración física. A veces, las radiografías pueden confirmar el diagnóstico y determinar la gravedad de la lesión. Sin embargo, en el caso de muchas personas que tienen una dislocación de hombro de grado bajo, hacerse radiografías en forma temprana suele ser normal. (Korsten, 2017, pág. 42)

La mayoría de las personas goza de una recuperación completa después de un tratamiento conservador. Una dislocación menor puede consolidarse en el lapso de unas semanas. La consolidación de una dislocación más grave puede tardar varias semanas o meses. Es posible que siempre tengas un bulto evidente en el hombro afectado; sin embargo, esto no debería alterar tu capacidad para usar ese hombro. (Mayo Clinic, 2018)

\section{Medicamentos}

Los analgésicos de venta libre, como el paracetamol (Tylenol u otros), el ibuprofeno (Advil, Motrin IB u otros) y el naproxeno sódico (Aleve), pueden ayudar a aliviar el dolor de hombro. (Korsten, 2017, pág. 42)

\section{Terapia}

- Descanso. Evita las actividades que agraven el dolor de hombro, en especial, cruzar el brazo afectado delante del cuerpo. Podrías querer inmovilizar el brazo en forma temporal con un cabestrillo para quitarle presión al hombro y ayudar a la curación.

- Hielo. El hielo puede reducir el dolor y la hinchazón del hombro. Utiliza una compresa fría de 15 a 20 minutos por vez.

- Fisioterapia. Los ejercicios de estiramiento y de fortalecimiento pueden ayudar a recuperar la fuerza y el movimiento del hombro. (Korsten, 2017, pág. 43)

No obstante, si el dolor persiste o si tienes una dislocación grave o una fractura de clavícula, la cirugía podría ser una opción. La cirugía puede volver a conectar los ligamentos desgarrados y reubicar o estabilizar los huesos lesionados.

\section{Conclusiones}

Las lesiones de la AAC son aproximadamente $12 \%$ de aquéllas que suceden en la cintura escapular. La luxación acromioclavicular (AC) representa $8 \%$ de todas las luxaciones en el cuerpo humano. La mayoría 
de estas lesiones se presentan en hombres, con una relación que va de 5 hasta 8:1 en comparación con las mujeres; la práctica deportiva es la primera causa de lesión.

En este tipo de lesión, los ligamentos acromioclaviculares, son aquellos que están adheridos a una cápsula articular y son muy finos. La estabilidad de la cápsula depende de los ligamentos coracoclaviculares, conoide y trapezoide. La lesión de los mismos, provoca una subluxación, pero la rotura completa de los 2 ligamentos coracoclaviculares es la verdadera causa de la luxación acromioclavicular.

Es allí, cuando el ligamento coracoacromial se inserta entre el borde supero externo de la apófisis coracoides y el vértice de la porción anterior del acromion. Como concepto, no es un "ligamento real", debido a que une 2 porciones del mismo hueso. Para su tratamiento, se observa la presencia de acuerdos existentes en cuanto a la implementación del tratamiento conservador en los grados I y II, no obstante, se observa existen grandes controversias en el tratamiento de las lesiones grado III.

El tratamiento de la articulación acromioclavicular sigue en debate debido a que no hay un consenso para el manejo óptimo de las lesiones en esta articulación. Rockwood y Green describieron dos escuelas fundamentales: 1) el tratamiento conservador o no quirúrgico y 2) el tratamiento quirúrgico. Aunque parece simple, es difícil elegir cuál es la mejor entre varias técnicas quirúrgicas para el tratamiento de la luxación AC.

De allí, que de manera general se establezca que, el tratamiento indicado en los casos de luxación acromioclavicular, específicamente el de los tipos I y II es conservador, en ellos, no se recomienda la cirugía, más bien, se realiza una inmovilización del hombro durante unas 3 semanas y posteriormente se inicia un periodo de recuperación de la movilidad del hombro. No obstante, para aquellas situaciones en que la luxación es de tipo III el tratamiento es más controvertido. De ahí, que sea importante valorar que a veces es difícil diferenciar radiográficamente una luxación acromioclavicular tipo III con la tipo V.

\section{Bibliografía}

Arias, F. (2010). Paradigmas de la Investigación Científica. España: Editorial: Luces.

Astudillo Valenzuela, C., \& Yoma Galleguillos, M. (2009). Descripción de la funcionalidad de hombro en pacientes operados artroscópicamente por inestabilidad anterior. Santiago de Chile: Universidad de Chile. Obtenido de http://repositorio. uchile.cl/bitstream/handle/2250/110558/Descripcion-de-la-funcionalidad-del-hombro.pdf?sequen$\mathrm{ce}=1 \&$ isAllowed $=\mathrm{y}$

Benhell, P. (s.f.). Biomechanical comparison.

Díaz Heredia, J., Ruiz Díaz, R., \& Valencia Mora, M. (2015). ¿Por qué tratamiento conservador de las luxaciones acromioclaviculares tipo iii? Revista Española de Artroscopia y Cirugía Articular, 22(1), 28-32. doi:10.1016/j.reaca.2015.06.001

Gutiérrez Blanco, M., Sánchez Bejarano, O., \& Reyes Casales, R. (2001). Estabilización dinámica de la luxación acromioclavicular grado III inmovilización con vepeaux invertido. Revista Archivo Médico de Camagüey, 5(1). Obtenido de http:// scielo.sld.cu/scielo. php?script=sci_arttext\&pi$\mathrm{d}=$ S1025-02552001000100003

Kayap, M. (2016). Acromioclavicular Joint Separation. Revista de Medicina General de la Habana Cuba, 11- 17.

Korsten, B. (2017). Acromioclavicular Third Degree Dislocation. Revista de Traumatología de la Universidad Autónoma Nacional de México, 33- 66.

Mayo Clinic. (2018). Hombro dislocado. Rochester, Minnesota, Estados Unidos: Mayo Clinic. Obtenido de https://www.mayoclinic.org/es-es/ diseases-conditions/separated-shoulder/diagnosis-treatment/drc-20354054

Sapunar, J., \& Velasco, M. (2000). Hidatidosis del omóplato con localización extraósea. Boletín chileno de parasitología, 55(3-4), 65-69. doi:10.4067/ S0365-94022000000300006

Slullitel, D., Galán, H., \& Della, V. (2016). Inestabilidad acromioclavicular. Tratamiento combinado artroscópico y miniinvasivo. Acta ortopédica mexicana, 30(1), 2-6. Obtenido de http://www. scielo.org.mx/scielo.php?script=sci_arttext\&pi$\mathrm{d}=$ S2306-41022016000100002 
Tamaoki, M., Lenza, M., \& Matsunaga, F. (2017). Intervenciones quirúrgicas versus conservadoras para el tratamiento de la luxación acromioclavicular del hombro en adultos. Cochrane, 12- 33. Obtenido de https://www.cochrane.org/es/CD007429/MUSKINJ_intervenciones-quirurgicas-versus-conservadoras-para-el-tratamiento-de-la-luxacion-acromioclavicular

\section{CITAR ESTE ARTICULO:}

León Portilla, V., Pico Segarra , J., \& Santillán Arias, M. (2020). Luxación acromioclavicular: tratamiento conservador. RECIMUNDO, 4(1), 267-276. doi:10.26820/recimundo/4.(1).enero.2020.267-276

(cC) $(1) \otimes(0)$

RECONOCIMIENTO-NOCOMERCIAL-COMPARTIRIGUAL CC BY-NC-SA

ESTA LICENCIA PERMITE A OTROS ENTREMEZCLAR, AJUSTARY Y CONSTRUIR A PARTIR DE SU OBRA CON FINES NO COMERCIALES, SIEMPRE Y CUANDO LE RECONOZCAN LA AUTORIIA Y SUS NUEVAS CREACIONES ESTÉN BAJO UNA LICENCIA CON LOS MISMOS TÉRMINOS. 